\title{
Immediate Effect of Trunk Flexion And Extension Isometric Exercise Using An External Compression Device On Electromyography of The Hip Extensor And Trunk Range of Motion Of Healthy Subjects
}

\section{Tian-Zong Huang}

Daejeon University

Suhn Yeop Kim ( $\boldsymbol{\sigma}$ kimsy@dju.kr)

Daejeon University https://orcid.org/0000-0002-0558-7125

\section{Research Article}

Keywords: Back extensor fatigue, electromyography, gluteus maximus, onset time, isometric exercise, trunk flexion ROM

Posted Date: December 28th, 2021

DOI: https://doi.org/10.21203/rs.3.rs-1179826/v1

License: (c) (i) This work is licensed under a Creative Commons Attribution 4.0 International License. Read Full License 


\section{Abstract}

Background: Low back pain (LBP) is a very common symptom experienced by individuals across all age groups. Previous study established that using a device known as Active Therapeutic Movement version 2 $\left(\right.$ ATM $^{\circledR} 2$ ) has been developed to improve pain and joint range of motion (ROM) in patients with LBP. However, no study has examined the physiological change in the muscle through ATM $^{\circledR} 2$-based exercise thus far. This study aimed to determine the immediate effects of $A T M^{\circledR} 2$ exercise on the contraction timing, back extension endurance, muscle fatigue, and trunk ROM of lumbar and lower limb muscles in healthy subjects.

Methods: Thirty-six healthy subjects (mean age $=23.16 \pm 2.3$ ) volunteered to participate in this study. Subjects were instructed to perform ROM test using sit and reach test, back extensor endurance test using Biering-Sorensen test, erector spinae (ES), lumbar multifidus (LM) fatigue and onset time of Gluteus maximus (GM) in prone hip extension using electromyography before and after trunk flexion and extension isometric exercises.

Results: The ROM in trunk flexion showed a significant increase by $7.9 \%$ after exercise compared to that before exercise $(\mathrm{p}<0.05)$. Relative $\mathrm{GM}$ contraction onset timing significantly decreased after exercise $(p<0.05)$. The result of the Sorensen test after exercise showed a trend of increase in duration time. Muscle fatigue in the LM, however, showed a significant increase $(p<0.05)$, whereas muscle fatigue in the ES was reduced without statistical significance.

Conclusions: The results base on this study showed a significant increase in the trunk ROM after trunk flexion and extension isometric exercise using an external compression device, while the relative contraction onset timing in the GM significantly reduced. Furthermore, the muscle endurance test after exercise showed a trend of increase in the duration time with a decreasing trend in muscle fatigue in the ES. Exercise based on $A T M^{\circledR} 2$ is an effective exercise protocol with an effect on biomechanics of healthy subjects. This exercise may be suitable in clinical practice for patients with LBP, for which long-term effects can be expected.

\section{Introduction}

Low back pain (LBP) is a very common symptom experienced by individuals across all age groups [1]. LBP is a symptom rather than a disease. As with other symptoms, such as headache or vertigo, there are numerous causes of LBP. The most common form of LBP is non-specific LBP, in which the pathological cause of pain cannot be determined [2]. According to a previous study, $85 \%$ patients in primary care exhibited non-specific LBP [3]. LBP is more common among women than among men and in the 40-69 years age group than in other age groups [2]. A cohort study conducted in 2009 showed that $42 \%$ patients with LBP had acute LBP that progressed to chronic LBP and approximately $11-12 \%$ patients had limitations in daily activities [4,5]. Chronic LBP is defined as continuous pain over 3 months, and among 
patients with LBP, approximately $10-20 \%$ have chronic LBP. With pain as a cause, patients with LBP experience health issues in terms of physical function and mental fatigue [6].

Simultaneous pain may be felt in multiple parts of the body in patients with LBP, who present greater number of physical and psychological health issues than healthy individuals without LBP [7]. LBP can be assessed using the visual analog scale, which measures subjective pain levels; questionnaire-based tests, such as Oswestry disability index, a functional test of the quality of life; radiographic diagnosis; and more objective physiological tests such as surface electromyography (SEMG) [8]. Many previous studies have shown significant differences in the SEMG activity between patients with LBP and healthy individuals, suggesting that SEMG could accurately distinguish the two groups of subjects. This verified the potential use of SEMG in the objective assessment of LBP [9]. In a study by Ambroz, Scott [10], the static standing posture and lumbar muscle activity on dynamic back flexion/extension exercise were compared between patients with LBP $(n=30)$ and healthy subjects $(n=30)$. The lumbar muscle activity was significantly higher in patients with LBP than in healthy subjects.

In addition, the form of electromyography (EMG) that applies variables extracted from the frequency spectrum was accepted as a method of objective assessment of lumbar muscle fatigue and recovery levels [11]. The EMG signal during isometric contraction was monitored through surface electrodes, and the median frequency of the spectrum was determined based on the number and type of primarily activated motor units. The median frequency is considered an objective scale of muscle fatigue because of it decreases with increasing muscle fatigue; in particular, the slope of the regression line of the timedependent central frequency is used as an indicator of the level of muscle fatigue [12]. Several protocols have been used to estimate muscle fatigue in patients with LBP. The easiest among them is the BieringSorensen test, where the subjects are guided to perform back extension exercise on a roman chair or laboratory table [13-16]. In a study by da Silva, Vieira [17], EMG analysis provided evidence of a significantly higher incidence of muscle fatigue in both young and old patients with chronic LBP than in healthy subjects. The results indicated a strong clinical significance of the lumbar muscle endurance test in LBP-related rehabilitation programs.

Prone hip extension (PHE) is generally performed during pathological examination of the waist area to assess stability or excess activity in the lumbopelvic region. In addition, PHE is applied in the evaluation of muscle contraction onset timing in the lumbopelvic region [18]. Bruno and Bagust [19] reported that, on $\mathrm{PHE}$, the muscle contraction onset timing of the gluteus maximus (GM) was substantially delayed in patients with LBP than in healthy subjects. Kwon and Koh [20] investigated the muscle contraction onset timing of the hamstring muscle (HM), erector spinae muscle (ES), and GM on PHE in patients with LBP and healthy subjects. The results showed that muscle contraction on PHE was the highest in the HM, followed by the ES and GM, in both groups. However, the contraction onset timing of the GM was significantly delayed compare to HM in the LBP group than in the healthy subject group.

Various intervention strategies are used for the management of LBP, ranging from surgical treatment to drug and non-drug therapies. Exercise therapy is the most widely applied method of conservation therapy, 
and the physical therapist generally prescribes the exercise intervention [21]. The most frequently used exercises for patients with LBP are McKenzie exercise, with a focus on lumbar extension exercise to enhance lumbar stability, and William exercise, mainly comprising the flexion exercise [22]. A device known as active therapeutic movement version 2 (ATM®2) (BackProject Corporation, San Jose, California) has been developed to improve pain and joint range of motion (ROM) in patients with LBP. The exercise intervention based on $A T M{ }^{\circledR} 2$ could reduce pain by fixing the hip and lower chest area with a belt. Before fixation active contraction along the direction (flexion or extension) that caused the pain, and then perform the maximum isometric contraction of the trunk exercise within the painless range. Consequently, exercise within the painless range and that restores the accurate position of the joint is likely to change muscle activity patterns controlled by the central nervous system to reduce pain caused during exercise [23]. Recently, there have been reported the results of applying ATM®2 in the treatment of patients with LBP in clinically. The level of pain reduced and the ROM increased after several minutes of exercise.

Previous studies regarding $A T M{ }^{\circledR} 2$ were conducted on patients with kyphoscoliosis or scoliosis $[24,25]$. In a case study by [23], where ATM ${ }^{2} 2$ was applied in an intervention, a significant improvement was reported when the pain level was measured at 4 and 8 weeks compared to that before intervention. However, limited and poor-quality evidence of the study that has examined the physiological change in the muscle through ATM ${ }^{\circledR}$ 2-based exercise thus far. Thus, this study aimed to determine the immediate effects of $A T M{ }^{\circledR} 2$-based exercise on the contraction onset timing of $\mathrm{GM}$, back extension endurance, back extensor muscle fatigue, and trunk ROM of lumbar in healthy subjects.

\section{Methods}

\section{Subjects}

The sample size was estimated in accordance with the objectives of this study using the Cohen's D equation in the $\mathrm{G}^{*}$ Power 3.1.9.2 program (University of Kiel, Kiel, Germany). We conducted a preliminary study on 10 subjects to maintain the testing power regarding the effects of ATM®2-based exercise, with the change in trunk flexibility before and after exercise as the primary indicator. Based on the data collected, the effect size was estimated at 0.65 , and by setting the level of significance at 0.05 and the testing power at 0.95 , the sample size was estimated to be $n=28$. Considering a $20 \%$ drop-out rate, a minimum of 36 individuals were recruited.

The principal investigator verbally explained the study purpose and procedures to all participants prior to the experiments. All subjects adequately understood and voluntarily agreed to participate in the study. The inclusion criteria were as follows: (1) an individual without pain in the waist or lower limbs; (2) an individual capable of lumbar extension on muscle endurance measurement without pain or discomfort; (3) an individual with no adverse reaction on maximum tension of the belt during long hours of ATM®2; and (4) an individual who understands the study purpose and voluntarily agreed to participate. The exclusion criteria were as follows: (1) an individual diagnosed with an orthopedic, neurological, 
cardiorespiratory, or mental disorder within the past 3 months; (2) an individual who sustained a wound in the waist or lower limb area within the past 6 months; and (3) an individual who requested discontinuation due to pain or discomfort during the experiments.

\section{Experimental procedures}

All subjects were requested to complete a questionnaire regarding the general characteristics and items related to the inclusion and exclusion criteria prior to the experiments. The selected subjects were then provided with explanations regarding the motions to be performed in the experiments, each of which were to be practiced at least three times for accurate understanding of the methods involved in each exercise, prior to the experiments. To measure the trunk ROM, the subjects performed sit and reach (SR) exercise, and the sitting trunk flexion tester was used. To measure the SEMG, SEMG electrodes were attached to the ES, lumbar multifidus muscle (LM), GM, and HM on the dominant side. The Biering-Sorensen test was used to measure lumbar extension endurance; however, to measure the GM and HM contraction onset timing, the subjects performed PHE. Isometric extension and flexion exercises were performed using $A T M{ }^{\circledR}$ 2. To set the performing order of isometric extension and flexion exercise for subjects, a randomizer (Research randomizer; http://www.randomizer.org/) was used prior to the experiments. All tests were performed before and after exercise to determine the immediate effects of each exercise. The experimental protocol was established, according to the ethical guidelines of the Helsinki Declaration and was approved by the Human Ethics Committee of Institutional Review Board (IRB) of Daejeon University (IRB No. 1040647-202110-HR-011-03) and subsequently registered in the Clinical Research Information Service (CRIS; KCT0006728). Written informed consent was obtained from individual or guardian participants.

\section{ATM $^{\circledR}$ 2-based exercise}

For $A T M{ }^{\circledR}$ 2-based exercise, subjects were instructed to perform trunk isometric flexion exercise or extension exercise in the standing posture (Fig. 1). The exercise direction was set to the one that elicited stronger pain at maximum flexion or maximum extension in the initial assessment. As this study included healthy subjects, both flexion and extension exercises were performed. Each subject wore two belts around the hip area and one belt around the chest area for fixation. The hip belt had two parts-upper and lower; the upper part was placed around the anterior superior iliac spine (ASIS), while the lower part was placed around the trochanter major. The chest belt was placed on the lower side of the chest. Belt compression was at a level that did not induce pain, and the maximum strength was controlled within a range that allowed stable breathing. Once the belts were in place, the subject was guided to perform extension exercise with the maximum isometric contraction in the trunk extension direction with hands held behind the head. For flexion exercise, the maximum isometric contraction was performed in the flexion direction as the subject held hands before the chest. In a single exercise, a 3-s isometric contraction was repeated 10 times, and a 2-s break was allowed between each contraction task.

\section{Lumbar extension endurance}


The Biering-Sorensen test, developed by Biering-Sorensen in 1984, was used to assess back extensor muscle endurance [26]. For the initial posture, the subject was guided to lay on the treatment table with the ASIS positioned at the edge of the table. The pelvis and legs were strapped in position using three belts in the areas close to the trochanter major, knees, and ankles. Prior to the test, the subject was allowed to rest the torso on a chair. The measurements were taken for as long as the subject could maintain the posture of the extension of the upper trunk away from the low chair and place the arms across the chest. The subject should maintain the posture in the neutral trunk alignment. The rater measured the time that the subject could maintain and remained beside the subject to provide assistance at the end of the test and during the break. The test was terminated when the subject could not maintain a posture of deviations greater than $10^{\circ}$ in the sagittal plane or when the duration time was $240 \mathrm{~s}$.

\section{Muscle fatigue}

To collect data on muscle activity, the SEMG device (TeleMyo 2400T, Noraxon, USA) was used to measure muscle activity and muscle contraction onset timing. A program with the designated software was used to analyze muscle activity of the GM, ES, and $\mathrm{HM}$ at the hip joint as well as the muscle contraction onset timing. The band pass filter, filtration filter, and rate of sample extraction were set at $20 \sim 450 \mathrm{~Hz}, 60 \mathrm{~Hz}$, and $1,024 \mathrm{~Hz}$, respectively. All collected data regarding muscle activity were analyzed using the root mean square (RMS) with a $50 \mathrm{~ms}$ moving window. Prior to electrode attachment, the target areas were shaved to minimize skin resistance and cleaned using an alcohol swab. A disposable $\mathrm{Ag} / \mathrm{AgCl}$ surface electrode was attached to each target area.

Muscle fatigue in the lumbar region was measured through EMG during endurance exercise. The measured areas were the LM and ES. For the LM, the electrode was attached to an area $3 \mathrm{~cm}$ away toward the exterior from the L5 spur. For the ES, the electrode was attached to an area $2 \mathrm{~cm}$ away toward both sides of the $L 2[27,28]$. EMG signals collected during the endurance test were analyzed based on the power spectrum through the fast Fourier transform. The overall gradient was estimated to obtain the resulting frequency data.

\section{Muscle contraction onset timing}

To measure the muscle contraction onset timing, data on the HM and GM on the dominant side on PHE were collected. The HM electrode was attached to an area in the middle, between the center of the knee, elbow, and femoral region [29]. The GM electrode was attached to an area in the middle, between the trochanter major and the sacrum, at $2 \mathrm{~cm}$ intervals on a diagonal line [30]. Hip joint extension was applied to the dominant leg. The subject was guided to lay in the prone position with the ankles aligned with the edges of the table on a neutral state. The arms were kept down in a comfortable position, while the back of the hand was kept upward in an anatomical posture. During hip joint extension, care was taken to prevent the legs from being adduction or abduction. All subjects were guided to ensure the knees are extension when performing hip joint extension. All measurements were repeated three times, and between each measurement, a 30-s break was given. The mean of the measurements was considered. 
Muscle contraction onset timing was analyzed using MyoReaseach Master Edition 1.06 XP software (Noraxon, Scottsdale, AZ, USA). The data based on muscle activity analysis were RMS treated, muscle contraction onset timing was analyzed using the designated program. Prior to PHE performance, the standard deviation (SD) for the mean of the EMG signal onset and the most stable 100-ms period were analyzed. The resulting values were applied in setting the relative muscle contraction onset timing as the first point of two consistent SD values above $25 \mathrm{~ms}$.

\section{Trunk flexion ROM}

The SR test was used to assess the trunk flexion ROM. The testing tool was installed as the subject sat on a flat surface in preparation for the reach motion. The subject was guided to take off the shoes and sit with both legs outstretched and both heels tightly touching the testing tool. The subject was guided to place hands overlapped on the testing tool for the reach, with both arms outstretched. At the signal from the rater, the subject breathed out and slowly bent the upper body, while pushing the scale with fingertips as far as they can go. A slow and steady motion was maintained to prevent the gauge of the tester from being pushed by bouncing, holding the end position for approximately 2 second. If the subject displayed inadequate understanding of the motion, the rater demonstrated the motion with the hands to induce the accurate motion. Any forced movements, such as excess push on the back or pull on the arms were prevented, and care was taken to prevent bending of the knees. Measurements were taken after an adequate level of practice. The values were recorded in centimeter for the maximum distance on the scale pushed by the subject as the upper body bent forward. The mean of duplicate measurements was considered for the final analysis.

\section{Data analysis}

Statistical analysis of all collected data was performed using the SPSS version 20.0 for Windows (SPSS Inc., Chicago, IL, USA). Descriptive statistics was applied to analyze the general characteristics of the subjects; the mean and standard deviation were calculated for all measured variables. The Shapiro-Wilk test was used to test the normality of the measured variables. The paired t-test was used to compare the changes in the trunk extension endurance, back extensor muscle fatigue, trunk ROM, and GM, HM contraction on PHE before and after exercise. The level of significance was set at $a=0.05$.

\section{Results}

\section{General characteristics of subjects}

Thirty-six subjects (22 men and 14 women) participated in this study, and the general characteristics, including the mean age, height, weight, and body mass index, are presented in Table 1. Across all measured variables, no sex differences were found; therefore, the data were analyzed without discrimination. 
Table 1

General characteristics of subjects $(n=36)$

\begin{tabular}{|c|c|}
\hline Variables & Mean \pm Standard deviation \\
\hline $\begin{array}{l}\text { Gender } \\
\text { (male/female) }\end{array}$ & $22 / 14^{\mathrm{a}}$ \\
\hline Age (year) & $23.16 \pm 2.30$ \\
\hline Height ( () & $169.67 \pm 9.89$ \\
\hline Weight ( () & $67.60 \pm 14.02$ \\
\hline BMI (『/ه) & $23.33 \pm 3.37$ \\
\hline
\end{tabular}

\section{Comparison of trunk flexion ROM before and after isometric exercise using $\mathrm{ATM}^{\circledR} 2$}

The ROM in trunk flexion was compared before and after isometric flexion and extension exercise using ATM $^{\circledR} 2$ (Table 2). The trunk ROM showed a significant increase by $7.9 \%$ after exercise compared to that before exercise $(p<0.05)$.

Table 2

Comparison of range of motion in trunk flexion before and after isometric exercise using ATM2 ( $n=36)$

\begin{tabular}{|lllllll|}
\hline Pre & & Post & t & Pvalue & Cohen's d \\
\hline $6.03 \pm$ & 13.45 & $9.18 \pm$ & 12.61 & -7.601 & $0.000^{*}$ & 0.24 \\
\hline \multicolumn{4}{|l}{ Values are presented as mean \pm standard deviation $(\mathrm{cm}),{ }^{\star} \mathrm{p}<0.05}$. \\
\hline
\end{tabular}

\section{Comparison of trunk muscle endurance before and after isometric exercise using $\mathrm{ATM}^{\circledR} 2$}

Trunk muscle endurance was compared before and after isometric flexion and extension exercise using ATM $^{\circledR} 2$ (Table 3). The result of the Biering-Sorensen test after exercise showed trend of increase in duration time, and muscle fatigue in the ES was reduced. Muscle fatigue in the LM, however, showed a significant increase $(p<0.05)$. 
Table 3

Comparison of duration time and fatigue of back muscle activation before and after isometric exercise using ATM2 ( $\mathrm{n}=36)$

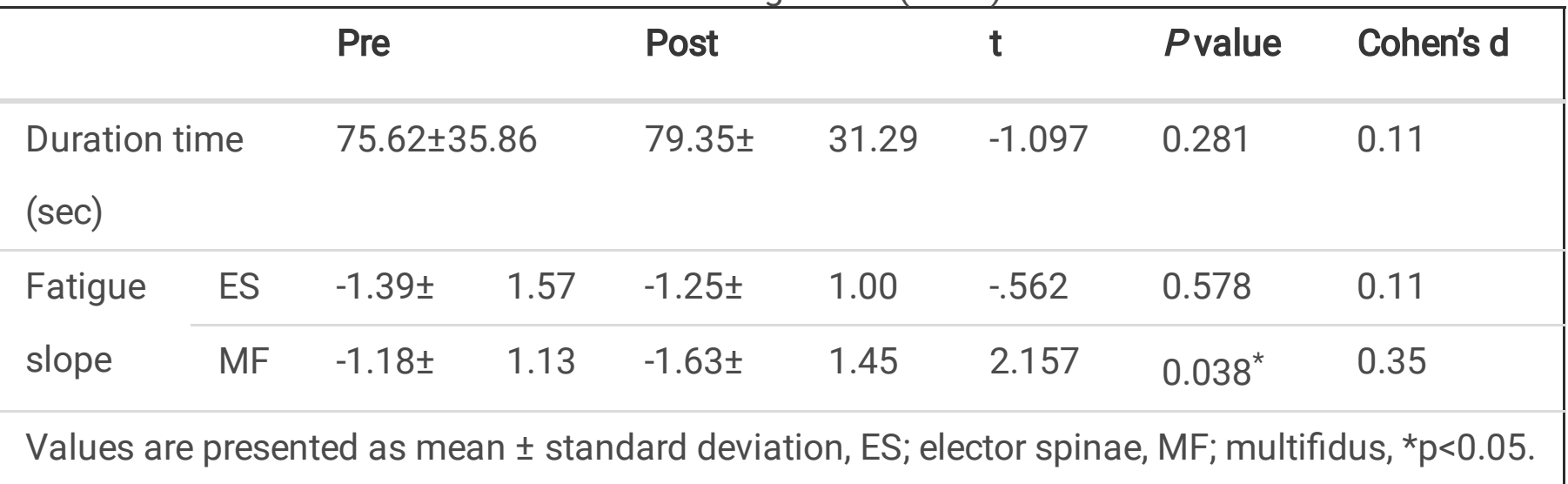

\section{Comparison of lower limb muscle contraction onset timing before and after isometric exercise using ATM $^{\circledR} 2$}

Table 4 presents the results of the comparison of the muscle contraction onset timing for trunk GM before and after isometric flexion and extension exercise using ATM $^{\circledR} 2$. The GM contraction onset timing was set as the relative timing in the analysis. The HM contraction onset timing was set as the reference (0), the time from HM contraction up to GM contraction was set as the negative (-), and the time of inadvance GM contraction before HM was set as the positive (+) when measuring the GM contraction onset timing. The results showed that the relative GM contraction onset timing significantly decreased after exercise $(p<0.05)$. Hence, there was a significant decrease in the interval between the GM and HM contraction onset timings after exercise.

Table 4

Comparison of onset time of gluteus maximus activation before and after isometric exercise using ATM2 ( $n=36)$

\begin{tabular}{|lllllll|}
\hline Pre & & Post & t & Pvalue & Cohen's d \\
\hline$-0.38 \pm$ & 0.37 & $-0.28 \pm$ & 0.30 & -2.216 & $0.033^{*}$ & 0.3 \\
\hline \multicolumn{7}{|l}{ Values are presented as mean \pm standard deviation $(\mathrm{sec}),{ }^{*} \mathrm{p}<0.05}$. \\
\hline
\end{tabular}

\section{Discussion}

This study was conducted to determine the immediate effects of isometric flexion and extension exercise with external compression through $\mathrm{ATM}^{\circledR} 2$ on lumbar extension endurance, back extensor muscle fatigue, lower limb muscle contraction onset timing, and trunk flexion ROM in healthy subjects. The results showed a significant increase in trunk flexion ROM after exercise, and a significant decrease in the relative GM contraction onset timing. For lumbar extension endurance, the Biering-Sorensen test showed 
trend of increase in the duration time on lumbar extension and muscle fatigue in the ES showed trend of decreased after exercise. Muscle fatigue in the LM, however, significantly increased.

The ATM ${ }^{\circledR} 2$ device has four belts for the safe fixation of the user's body onto a vertical treatment table. The belt strength can be controlled for different exercises at various angles. In a case report on a 55-yearold patient with frozen shoulders caused by kyphoscoliosis, the effects of the exercise therapy based on ATM $^{\circledR} 2$ were examined. The subject showed recovery of an almost normal ROM on both shoulders, and the kyphoscoliosis posture improved after the intervention of a few weeks [24]. Lewis, Diaz [25] investigated the effects of the exercise intervention using a program with ATM $^{\circledR} 2$ and home exercise for 4 weeks in 43 patients diagnosed with scoliosis. The results showed an increase in the spinal ROM after intervention, with improvements in pelvic alignment, pain, and disability. Patients with LBP have reduced lumbar ROM and proprioception with slower motion than healthy individuals, [31]. Thus, therapists have used various treatment methods to increase lumbar flexibility. Mazloum, Sahebozamani [32] reported a significant increase in the trunk flexion ROM through a training based on pilates and extension exercise that significantly reduced pain in patients with LBP. In clinical practice, the use of $A T M^{\circledR} 2$ in patients with LBP requires the belt tension strength to be set based on the limited ROM that pain provocation when performing trunk flexion or extension. As this study targeted healthy adult individuals, the belt tension strength during exercise was set to a maximum level without causing discomfort. Ito and Gamada [33] evaluated the trunk ROM using the finger-to-floor distance of healthy adult men between the group who performed $\mathrm{ATM}^{\circledR}$ 2-based exercise and the control group after intervention. The results showed that the ROM significantly increased in the exercise group than in the control group. Nejishima, Sugiura [23] also examined the effects of $A T M{ }^{\circledR}$ 2-based exercise in 14 patients with LBP and reported that the level of pain and LBP disability (Roland-Morris Disability Questionnaire) had significantly decreased after exercise during the tests at week 4 or 8 . Likewise, in this study, the SR test after exercise showed a significant increase in the trunk flexion ROM. The increase in trunk ROM through exercise suggested a potential intervention effect of the exercise on patients with LBP.

Lumbopelvic stability is considered a critical factor in the prevention and treatment of injury based on the potential contribution to recovery from injury and subsequent improvement [34]. For humans to maintain a straight posture, it is necessary to produce a force against gravity. The weights of the upper limbs, trunk, and head are conveyed to the pelvis via the spine. To maintain stability in the pelvis, the force of compression should be increased [35]. Most treatments for patients with LBP include trunk muscle strengthening training, and recently, core stabilization exercise has frequently been applied [36]. In addition, an external compression device may be used to provide stability. Arumugam, Milosavljevic [37] reported that the external compression on the pelvis enhanced the form closure of the sacroiliac joint as well as the force closure and exercise control through reduced compensation on lumbar stability muscles. In another previous study, the use of a pelvic compression belt had an effect on the thickness of the LM and ES have been proved through ultrasound. Its potential use as an effective assistive tool in the muscle strengthening exercise [38]. The $\mathrm{ATM}^{\circledR} 2$ device in this study may provide a pelvic compression effect 
during the isometric exercise. The application of the device on patients with LBP is anticipated to produce more effective therapeutic effects than the common muscle strengthening exercise.

The lumbar region has an important dynamic function of supporting the upper trunk while conveying the compression and shearing forces that arise during daily activities to the lower limbs [39]. This provides spinal stability that is maintained based on the passive support from the bone and ligament structure, active support from the muscles, and interactions among the control systems via the central nervous system [40]. Previous studies reported that, in patients with LBP, the limb or trunk exercise led to a different type of exercise control from that in healthy subjects [41-43]. In patients with LBP, compared with healthy subjects, the trunk muscle activity and the order of contraction onset timing changed [44]. Causes spinal movement involved multi-segment joints, the limitation of a single-segment to increase movement of another segment in compensation, and movement of the limbs is related to the movement of the spine [20]. Janda and Jull [45] reported that on PHE, the normal order of muscle activity is the contraction of the GM preceding the contraction of the $\mathrm{HM}$ and that the change in this order would induce excess compensatory lumbar extension, thus resulting in weakening of the GM. In constrast, Pierce and Lee [46] reported that on hip joint extension, the order of muscle contraction was consistently initiated by the HM prior to extension. Kwon and Koh [20] also reported that on PHE or hip joint extension in a standing posture, the HM began contraction first prior to the GM. Similarly, in this study, GM contraction preceded HM contraction before and after exercise in two subjects, while all other subjects showed the HM contraction first. Kwon and Koh [20] reported that on PHE, GM contraction was significantly delayed compared to HM contraction in the LBP group; the delay was by $0.03 \mathrm{~s}$ in the control group and $0.18 \mathrm{~s}$ in the LBP group. The measurements for PHE in this study showed that the mean delay in GM compare to $\mathrm{HM}$ was $0.38 \mathrm{~s}$ before exercise and $0.28 \mathrm{~s}$ after exercise, with a significant difference in muscle contraction onset timing before and after exercise. For PHE, it is easy for the HM to be overused when it becomes the dominant extensor as the GM is unable to function adequately, which can ultimately lead to LBP [47]. In a study by Hungerford, Gilleard [48], patients with sacroiliac joint pain were guided to stand on one leg and perform the hip flexion on the other leg and as the biceps muscle contraction occurred first, a delay in the GM contraction was observed. Thus, it is presumed that the adjustment of the order of muscle activity has a critical role in the prevention and treatment of LBP.

The spine itself is an unstable structure with appropriate support from the surrounding muscles and tissues for compensation [49]. Regarding muscle endurance in the lumbar region, muscle fatigue is a frequently detected problem in patients with LBP and a risk factor of LBP [50-52]. Thus, the state of the trunk can be accurately determined through the assessment of lumbar muscle function and fatigue to provide suitable treatments. The Biering-Sorensen test in this study was used to assess the muscle endurance around the lumbar region based on the duration time and EMG. In a study by Jung, Lee [53], the group that received the 4-week manual therapy and the group that received exercise therapy showed reduced pain after intervention, while trunk stability and duration time in the Biering-Sorensen test increased. This study hypothesized that the duration time in the Biering-Sorensen test would increase after exercise to decrease muscle fatigue due to test motions. The results of this study showed a trend of increase, despite the lack of significance, in duration time after exercise. In addition, muscle fatigue in the 
ES decreased, although without statistical significance. However, contrary to the hypothesis, muscle fatigue in the LM increased after exercise. This was attributed to the difficulty in performing test motions repeatedly within a short period of time between two measurements, despite the adequate resting time. Furthermore, in the Biering-Sorensen test used in this study, the subjects were guided to maintain posture up to as high as $120 \mathrm{~s}$. For some subjects who could go beyond $120 \mathrm{~s}$, the experiment ended as they reached 120 s to prevent potential influence on the statistical results. In addition, the aim of this study was to analyze the immediate effects of exercise intervention and performing a single exercise might have prevented the predicted effects from being reached. In future, the effects of a long-term exercise intervention with a 2- or 4-week training program in terms of muscle endurance and muscle fatigue should be investigated.

This study has several limitations. First, only the immediate effects of ATM@2-based isometric flexion and extension exercise were evaluated. Second, the subjects were not patients with LBP, but healthy individuals. Third, the results may have been influenced by subjective influencing factors during the procedures as they could not be completely excluded. In future studies, these limitations should be compensated to procure more scientifically valid clinical data.

\section{Conclusion}

This study investigated the immediate effects of isometric flexion and extension exercise on the contraction onset timing, back extension endurance, muscle fatigue, and trunk ROM of the lumbar and lower limb areas in healthy subjects. Exercise was performed using ATM ${ }^{\circledR} 2$ with external compression on the hip area for fixation. The results showed a significant increase in the trunk ROM after exercise, while the relative contraction onset timing in the lower limb GM significantly reduced. Furthermore, the muscle endurance test after exercise showed a trend of increase in the duration time with a decreasing trend in muscle fatigue in the ES.

In conclusion, isometric exercise based on $\mathrm{ATM}^{\circledR} 2$ is an effective exercise protocol with an effect on biomechanics of healthy subjects. This study provides a good starting point for discussion and further research for which long-term effects of the exercise. This exercise might be applied in clinical practice for patients with LBP.

\section{Abbreviations}

ASIS: Anterior superior iliac spine; ATM ${ }^{@} 2$ : active therapeutic movement version 2; CRIS: clinical research information service; ES: Erector spinae; GM: Gluteus maximus; HM: Hamstring; IRB: institutional review board; LBP: low back pain; LM: Lumber multifidus; PHE: Prone hip extension; RMS: Root mean square; ROM: Range of motion; SD: Standard deviation; SEMG: Surface electromyography; SR: Sit and reach.

\section{Declarations}




\section{Ethical approval and consent to participate}

The experimental protocol was established, according to the ethical guidelines of the Helsinki Declaration and was approved by the Human Ethics Committee of Institutional Review Board (IRB) of Daejeon University (IRB No. 1040647-202110-HR-011-03) and subsequently registered in the Clinical Research Information Service (CRIS; KCT0006728)

\section{Consent for publication}

Witten consent for the use of the photos of ATM@2-based exercises was obtained

\section{Availability of data and materials}

Not applicable

\section{Competing interests}

Not applicable.

\section{Funding}

Not applicable.

\section{Informed consent}

Written informed consent was obtained from individual or guardian participants.

\section{Authors' contribution}

Huang and Kim made both the conception and the design of the work and drafted the first version of the manuscript. Huang collected data and did the statistical analysis. Each author made substantial contributions to the design of the work as well as to the acquisition, analysis, and interpretation of data.

\section{Acknowledgements}

Not applicable.

\section{References}

1. Hartvigsen J, Hancock MJ, Kongsted A, Louw Q, Ferreira ML, Genevay S, et al. What low back pain is and why we need to pay attention. The Lancet. 2018;391(10137):2356-67. http://dx.doi.org/10.1016/S0140-6736(18)30480-X.

2. Maher $C$, Underwood $M$, Buchbinder R. Non-specific low back pain. The Lancet. 2017;389(10070):736-47. http://dx.doi.org/10.1016/S0140-6736(16)30970-9. 
3. Koes BW, Van Tulder M, Lin CWC, Macedo LG, McAuley J, Maher C. An updated overview of clinical guidelines for the management of non-specific low back pain in primary care. Eur Spine J. 2010;19(12):2075-94. http://dx.doi.org/10.1007/s00586-010-1502-y.

4. Costa LCM, Maher CG, McAuley JH, Hancock MJ, Herbert RD, Refshauge KM, et al. Prognosis for patients with chronic low back pain: inception cohort study. Bmj. 2009;339

http://dx.doi.org/10.1136/bmj.b3829.

5. Airaksinen O, Brox JI, Cedraschi C, Hildebrandt J, Klaber-Moffett J, Kovacs F, et al. European guidelines for the management of chronic nonspecific low back pain. Eur Spine J. 2006;15(Suppl 2):s192.

6. Maher CG, Latimer J, Hodges PW, Refshauge KM, Moseley GL, Herbert RD, et al. The effect of motor control exercise versus placebo in patients with chronic low back pain. BMC Musculoskel Dis. 2005;6(1):1-8.

7. Hartvigsen J, Natvig B, Ferreira M. Is it all about a pain in the back? Best Pract Res Cl Rh. 2013;27(5):613-23. http://dx.doi.org/10.1016/j.berh.2013.09.008.

8. Yoo DJ, Cho JH, Chung SH. A review of studies comparing of surface electromyography values between the low-back pain group and healthy controls. Journal of Korean Medicine Rehabilitation. 2013;23(4):83-93.

9. Geisser ME, Ranavaya M, Haig AJ, Roth RS, Zucker R, Ambroz C, et al. A meta-analytic review of surface electromyography among persons with low back pain and normal, healthy controls. The journal of pain. 2005;6(11):711-26. http://dx.doi.org/10.1016/j.jpain.2005.06.008.

10. Ambroz C, Scott A, Ambroz A, Talbott EO. Chronic low back pain assessment using surface electromyography. J Occup Environ Med. 2000;42(6):660-9.

11. De Luca CJ. Use of the surface EMG signal for performance evaluation of back muscles. Muscle \& Nerve: Official Journal of the American Association of Electrodiagnostic Medicine. 1993;16(2):210-6. http://dx.doi.org/10.1002/mus.880160216.

12. De Luca CJ. The use of surface electromyography in biomechanics. J Appl Biomech. 1997;13(2):135-63. http://dx.doi.org/10.1123/jab.13.2.135.

13. Massoud Arab A, Salavati M, Ebrahimi I, Ebrahim Mousavi M. Sensitivity, specificity and predictive value of the clinical trunk muscle endurance tests in low back pain. Clin Rehabil. 2007;21(7):640-7. http://dx.doi.org/10.1177/0269215507076353.

14. Biering-Sørensen F. Physical measurements as risk indicators for low-back trouble over a one-year period. Spine. 1984;9(2):106-19. http://dx.doi.org/10.1097/00007632-198403000-00002. 
15. Larivière C, Da Silva RA, Arsenault AB, Nadeau S, Plamondon A, Vadeboncoeur R. Specificity of a back muscle roman chair exercise in healthy and back pain subjects. Med Sci Sport Exer. 2011;43(1):15764. http://dx.doi.org/10.1249/mss.0b013e3181e96388.

16. da Silva Jr RA, Arsenault AB, Gravel D, Larivière $C$, de Oliveira Jr E. Back muscle strength and fatigue in healthy and chronic low back pain subjects: a comparative study of 3 assessment protocols. Arch Phys Med Rehab. 2005;86(4):722-9. http://dx.doi.org/10.1016/j.apmr.2004.08.007.

17. da Silva RA, Vieira ER, Cabrera M, Altimari LR, Aguiar AF, Nowotny AH, et al. Back muscle fatigue of younger and older adults with and without chronic low back pain using two protocols: a case-control study. J Electromyogr Kines. 2015;25(6):928-36. http://dx.doi.org/10.1016/j.jelekin.2015.10.003.

18. Suehiro T, Mizutani M, Ishida H, Kobara K, Osaka H, Watanabe S. Individuals with chronic low back pain demonstrate delayed onset of the back muscle activity during prone hip extension. J Electromyogr Kines. 2015;25(4):675-80. http://dx.doi.org/10.1016/j.jelekin.2015.04.013.

19. Bruno PA, Bagust J. An investigation into motor pattern differences used during prone hip extension between subjects with and without low back pain. Clinical Chiropractic. 2007;10(2):68-80. http://dx.doi.org/10.1016/j.clch.2006.10.002.

20. Kwon OY, Koh EK. The comparison of the onset time of hamstring, gluteus maximus, and lumbar erector spinae muscle activity during hip extension between subjects with low back pain and healthy subjects. Physical Therapy Korea. 2002;9(2):33-42.

21. Van Middelkoop M, Rubinstein SM, Verhagen AP, Ostelo RW, Koes BW, van Tulder MW. Exercise therapy for chronic nonspecific low-back pain. Best Pract Res Cl Rh. 2010;24(2):193-204. http://dx.doi.org/10.1016/j.berh.2010.01.002.

22. Lee HK, Cho YH, Lee JC. The effect of improve the waist flexibility, the waist muscular strength and the waist balance which grafted in William \& Mckenzie exercise with swiss ball. Journal of Korean Society of Physical Medicine. 2013;8(4):479-87. http://dx.doi.org/10.13066/kspm.2013.8.4.479.

23. Nejishima M, Sugiura T, Kubo Y, Kobori K, Gamada K, Yokoyama S, et al. the effect of an exercise using an active therapeutic movement device on mechanical low back pain: A case series study. Rigakuryoho Kagaku. 2011;26(2):255-30.

24. Lewis C, Erhard R, Drysdale G. Kyphoscoliosis improvement while treating a patient for adhesive capsulitis using the active therapeutic movement version 2. J Manip Physiol Ther. 2008;31(9):715-22. http://dx.doi.org/10.1016/j.jmpt.2008.10.003.

25. Lewis C, Diaz R, Lopez G, Marki N, Olivio B. A preliminary study to evaluate postural improvement in subjects with scoliosis: active therapeutic movement version 2 device and home exercises using the 
Mulligan's mobilization-with-movement concept. J Manip Physiol Ther. 2014;37(7):502-9. http://dx.doi.org/10.1016/j.jmpt.2014.07.005.

26. Latimer J, Maher CG, Refshauge K, Colaco I. The reliability and validity of the Biering-Sorensen test in asymptomatic subjects and subjects reporting current or previous nonspecific low back pain. Spine. 1999;24(20):2085.

27. Ahn CS, Kim HS, Kim MC. The effect of the EMG activity of the lower leg with dynamic balance of the recreational athletes with functional ankle instability. J Phys Ther Sci. 2011;23(4):579-83. http://dx.doi.org/10.1589/jpts.23.579.

28. Fullam K, Caulfield B, Coughlan GF, Delahunt E. Kinematic analysis of selected reach directions of the Star Excursion Balance Test compared with the Y-Balance Test. J Sport Rehabil. 2014;23(1):27-35. http://dx.doi.org/10.1123/JSR.2012-0114.

29. Oh Dw. effects of shoe heel height on walking velocity and electromyographic activities of lower extremity muscles during short-and long-distance walking in young females. Physical Therapy Korea. 2019;26(2):16-23. http://dx.doi.org/10.12674/ptk.2019.26.2.016.

30. Oh JS, Cynn HS, Won JH, Kwon OY, Yi CH. Effects of performing an abdominal drawing-in maneuver during prone hip extension exercises on hip and back extensor muscle activity and amount of anterior pelvic tilt. J Orthop Sport Phys. 2007;37(6):320-4. http://dx.doi.org/10.2519/jospt.2007.2435.

31. Laird RA, Gilbert J, Kent P, Keating JL. Comparing lumbo-pelvic kinematics in people with and without back pain: A systematic review and meta-analysis. BMC Musculoskel Dis. 2014;15(1):1-13. http://dx.doi.org/10.1186/1471-2474-15-229.

32. Mazloum V, Sahebozamani M, Barati A, Nakhaee N, Rabiei P. The effects of selective Pilates versus extension-based exercises on rehabilitation of low back pain. Journal of bodywork and movement therapies. 2018;22(4):999-1003. http://dx.doi.org/10.1016/j.jbmt.2017.09.012.

33. Ito $K$, Gamada K. The immediate effects of therapeutic exercise using the ATM ${ }^{\circledR} 2$ on trunk and hip range of motion of healthy male individuals: A randomized controlled study. Rigakuryoho Kagaku. 2014;29(5):735-8.

34. Willardson JM. Core stability training: applications to sports conditioning programs. The Journal of Strength \& Conditioning Research. 2007;21(3):979-85.

35. Vleeming A, Schuenke M. Form and force closure of the sacroiliac joints. PM\&R. 2019;11:24-31. http://dx.doi.org/10.1002/pmrj.12205.

36. Shamsi MB, Rezaei M, Zamanlou M, Sadeghi M, Pourahmadi MR. Does core stability exercise improve lumbopelvic stability (through endurance tests) more than general exercise in chronic low back 
pain? A quasi-randomized controlled trial. Physiotherapy Theory and Practice. 2016;32(3):171-8. http://dx.doi.org/10.3109/09593985.2015.1117550.

37. Arumugam A, Milosavljevic S, Woodley S, Sole G. Effects of external pelvic compression on form closure, force closure, and neuromotor control of the lumbopelvic spine-A systematic review. Man Ther. 2012;17(4):275-84. http://dx.doi.org/10.1016/j.math.2012.01.010.

38. Baek IC, Shim JH. Pelvic Compression belt convergence impact on the thickness of multifidus and erector spinae muscles. Journal of the Korea Convergence Society. 2016;7(5):51-7. http://dx.doi.org/10.15207/JKCS.2016.7.5.051.

39. Cholewicki J, McGill SM. Mechanical stability of the in vivo lumbar spine: implications for injury and chronic low back pain. Clin Biomech. 1996;11(1):1-15. http://dx.doi.org/10.1016/0268-0033(95)00035-6.

40. Panjabi MM. The stabilizing system of the spine. Part I. Function, dysfunction, adaptation, and enhancement. J Spinal Disord Tech. 1992;5:383-9.

41. Hodges PW, Richardson CA. Inefficient muscular stabilization of the lumbar spine associated with low back pain: a motor control evaluation of transversus abdominis. Spine. 1996;21(22):2640-50.

42. Hodges PW, Richardson CA. Contraction of the abdominal muscles associated with movement of the lower limb. Physical therapy. 1997;77(2):132-42.

43. Hodges PW, Richardson CA. Altered trunk muscle recruitment in people with low back pain with upper limb movement at different speeds. Arch Phys Med Rehab. 1999;80(9):1005-12.

44. O'Sullivan PB, Twomey L, Allison GT. Altered abdominal muscle recruitment in patients with chronic back pain following a specific exercise intervention. J Orthop Sport Phys. 1998;27(2):114-24. http://dx.doi.org/10.2519/jospt.1998.27.2.114.

45. Janda V, Jull G. Muscles and motor control in low back pain: Assessment and management. Physical Therapy of the Low Back. Churchill livingstone. 1987:253-78.

46. Pierce MN, Lee WA. Muscle firing order during active prone hip extension. J Orthop Sport Phys. 1990;12(1):2-9. http://dx.doi.org/10.2519/jospt.1990.12.1.2.

47. Sahrmann S. Diagnosis and treatment of movement impairment syndromes: St Louis: Mosby; 2002. 51-108 p.

48. Hungerford B, Gilleard W, Hodges P. Evidence of altered lumbopelvic muscle recruitment in the presence of sacroiliac joint pain. Spine. 2003;28(14):1593-600.

49. Van Dieën JH. Asymmetry of erector spinae muscle activity in twisted postures and consistency of muscle activation patterns across subjects. Spine. 1996;21(22):2651-61. 
50. Hultman G, Nordin M, Saraste H, Ohlsen H. Body composition, endurance, strength, cross-sectional area, and density of MM erector spinae in men with and without low back pain. J Spinal Disord Tech. 1993;6(2):114-23.

51. Moffroid MT. Endurance of trunk muscles in persons with chronic low back pain: Assessment, performance, training. J Rehabil Res Dev. 1997;34:440-7.

52. Robinson ME, Cassisi JE, O'Connor PD, MacMillan M. Lumbar iEMG during isotonic exercise: Chronic low back pain patients versus controls. J Spinal Disord Tech. 1992;5(1):8-15. http://dx.doi.org/10.1097/00002517-199203000-00002.

53. Jung YD, Lee HO, Song MY. The effect of home exercise program on pelvic alignment and lumbar scoliosis of chronic low back pain. Journal of the Korean Society of Physical Medicine. 2009;4(3):133-40.

\section{Figures}

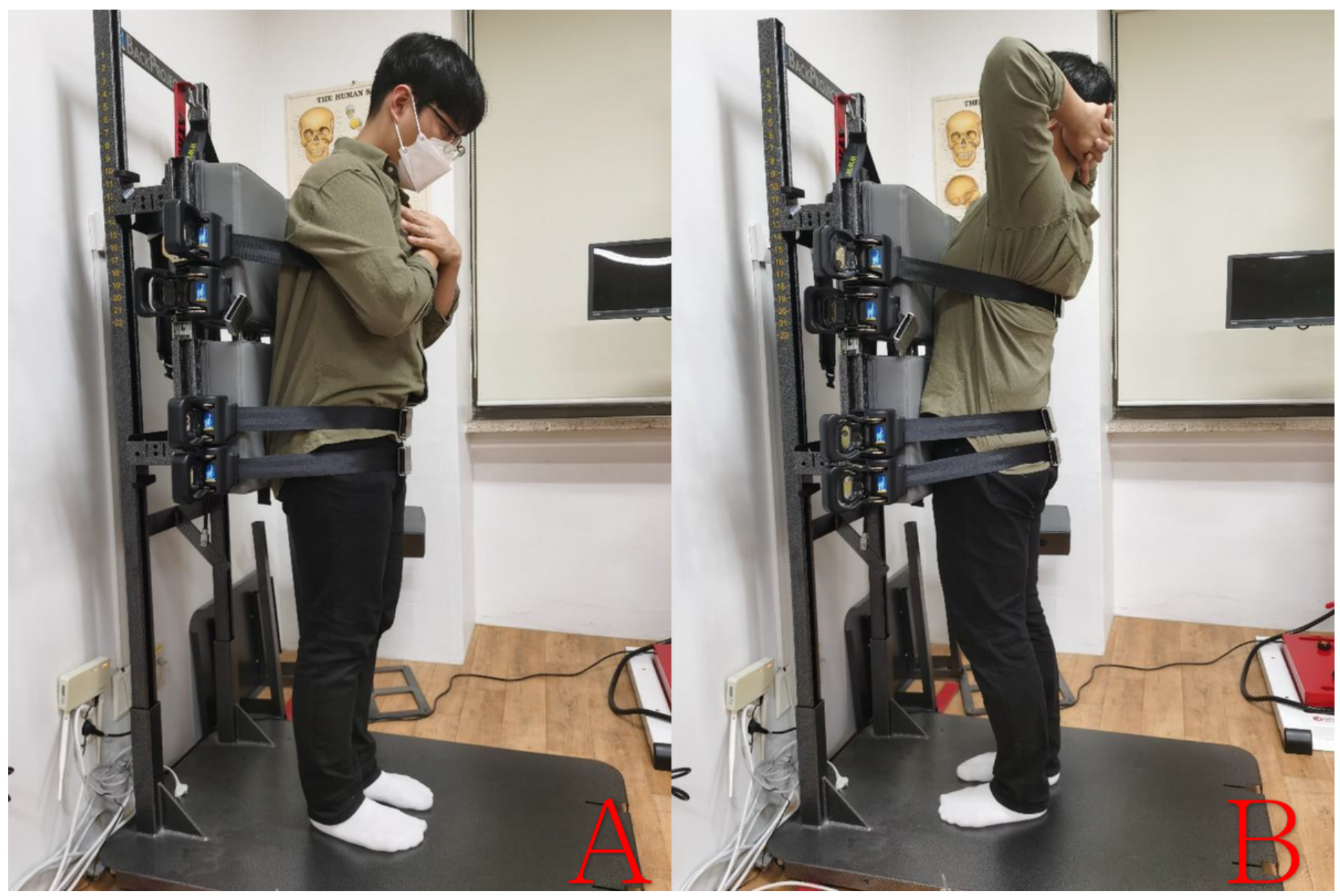

Figure 1 
Isometric flexion and extension exercise using ATM ${ }^{\circledR} 2$. A: isometric flexion exercise; B: isometric extension exercise. 